\title{
EFECTOS DEL FUEGO EN EL COMPORTAMIENTO HIDROLÓGICO DE DOS CUENCAS EN EL PREPIRINEO ARAGONÉS (ESPAÑA)
}

\author{
M.T. ECHEVERRÍA ARNEDO, P. IBARRA BENLLOCH \\ M. SEBASTIÁN LÓPEZ y F. PÉREZ CABELLO. \\ Departamento de Geografía y Ordenación del Territorio. Universidad de Zaragoza. \\ Pedro Cerbuna, 12, 50009-Zaragoza \\ Correo electrónico de contacto: mtecheve@unizar.es
}

\begin{abstract}
RESUMEN: El comportamiento hidrológico de dos cuencas forestales afectadas por un fuego forestal. El diferente porcentaje de superficie quemada en ambos espacios se traduce en un distinto comportamiento hidrológico, analizado mediante hidrogramas de crecida y un análisis estadístico comparativo. En la cuenca quemada, la respuesta de caudal a la precipitación es más rápida y abundante, en relación con una mayor descarga superficial ligada a una menor infiltración del agua en el suelo (coeficientes de escorrentía: $10 \%$ comparado con el $4.8 \%$ obtenido en la cuenca no quemada).
\end{abstract}

ABSTRACT: The hydrological behaviour of two basins affected by a forest fire. The different percentage of burnt surface in both areas is translated in a different hydrological behaviour, analysed through the discharge hydrograms and a comparative statistical analysis. In the burnt catchment area, the hydrological response to the precipitation is bigger and faster than in the other basin, in relation to a greater superficial runoff and a smaller infiltration rate (mean runoff coefficients: $10 \%$ compared to the $4.8 \%$ obtained at the non-burnt catchment).

Palabras clave: Fuego forestal, comportamiento hidrológico, caudal, precipitación, escorrentía, Pre-Pirineos, España.

Key words: Forest fire, hydrological behaviour, water discharge, rainfall, runoff, PrePyrenees, Spain. 


\section{Introducción}

Los incendios forestales constituyen uno de los mayores impactos ambientales en el entorno mediterráneo (Trabaud, 1981). Las consecuencias medioambientales de los fuegos se dejan notar no sólo en la vegetación, sino en los suelos afectados por el incendio (Shakesby y Doerr, 2006), modificando su estabilidad estructural (Molina et al., 1994, Martínez Fernández y Díaz Pereira, 1994, Fernández et al., 2005) y, por lo tanto, su susceptibilidad a la erosión (De Bano et al., 1979, Díaz-Fierros et al., 1987, DíazFierros y Rubio, 1990, Coelho et al., 1990, Soler y Sala, 1992, Sala y Rubio,1994, Soler et al., 1994, Kutiel et al., 1995, Úbeda y Sala, 1998) y su comportamiento hidrológico, al reducir tanto la cantidad de materia orgánica (Giovannini y Lucchesi, 1983, Sánchez et al., 1994), como la tasa de infiltración (DeBano et al., 1970, DeBano, 1971, DíazFierros et al., 1987, Marqués y Mora, 1992, Calvo-Cases y Cerdà, 1994, Soto et al., 1994, Cerdà et al, 1995, Bissonais, 1996, Cerdà et al., 1998, Pérez-Cabello et al., 2003 a y b, Strunk, 2005).

El caso español es especialmente grave, ya que en las últimas décadas el fuego incontrolado es la principal causa de deforestación de los bosques mediterráneos con una superficie quemada que puede alcanzar algunos años las 500.000 ha. En la Comunidad Autónoma de Aragón los incendios son uno de los principales problemas que afectan a las masas forestales del territorio. Este hecho provoca importantes cambios en el comportamiento hidrológico de las cuencas fluviales, a partir de la degradación de la cubierta vegetal y los cambios en las características físico-químicas del suelo derivando en modificaciones de los coeficientes de escorrentía e infiltración. Por otro lado, las labores que siguen al fuego (saca de madera, repoblaciones forestales), pueden reactivar ciertos procesos de degradación edáfica, tales como la pérdida de estabilidad y de materia orgánica, así como la compactación de los horizontes superiores.

En el presente trabajo se pretende caracterizar el comportamiento hidrológico, a través del estudio de hidrogramas de crecida, de dos cuencas prepirenaicas contiguas con características ambientales similares y afectadas por un incendio donde posteriormente se efectuaron labores de saca de madera y repoblación forestal.

\section{2. Área de estudio}

El área de estudio se sitúa en la umbría de la Sierra de San Juan de la Peña (Prepirineo Oscense), concretamente en la cabecera del barranco de Alastuey (Fig. 1).

El clima de la zona es de tipo submediterráneo/subhúmedo. La precipitación media anual es de unos $850 \mathrm{~mm}$. Presenta un régimen pluviométrico equinoccial con máximos principales en primavera y cierto déficit hídrico en el verano, que frecuentemente queda interrumpido por fenómenos tormentosos. La temperatura media es de $11^{\circ} \mathrm{C}$. Los veranos son calurosos y en los meses invernales el riesgo de heladas es fuerte (temperatura media de las mínimas inferior a $0^{\circ} \mathrm{C}$ ). 
La vegetación, previa al fuego, estaba constituida, en la cuenca afectada por el incendio, por un pinar denso de Pinus sylvestris con un sotobosque compuesto por Buxus sempervirens, Coronilla emerus, y Brachypodium sp, como especies dominantes.

Desde el punto de vista edáfico, en los depósitos de ladera asentados sobre estratos alternantes de areniscas y lutitas eo-oligocenas, se reconocen cambisoles eutricos y calcáricos, bien drenados y estructurados, con espesores en torno a 75-100 cm, que superficialmente presentan distintos grados de alteración en función de los efectos del fuego y de la actividad antrópica posterior al mismo.

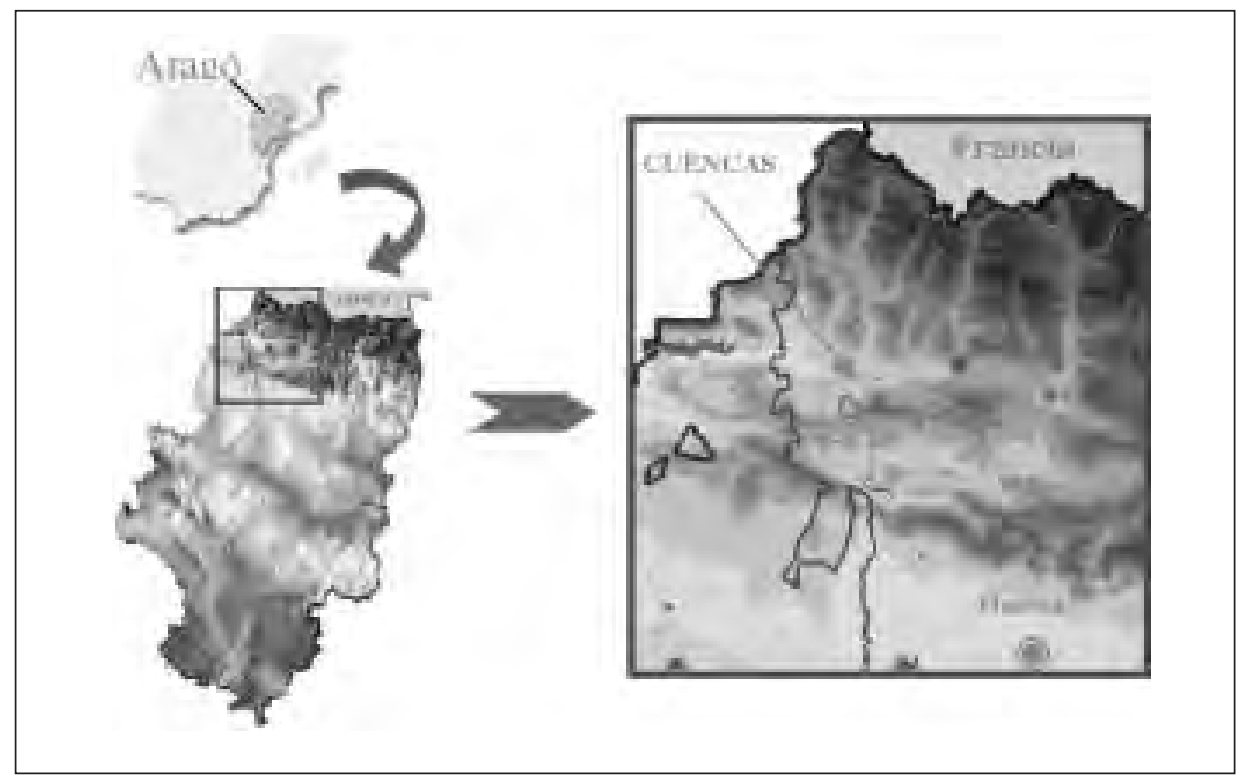

Figura 1. Localización del área de estudio.

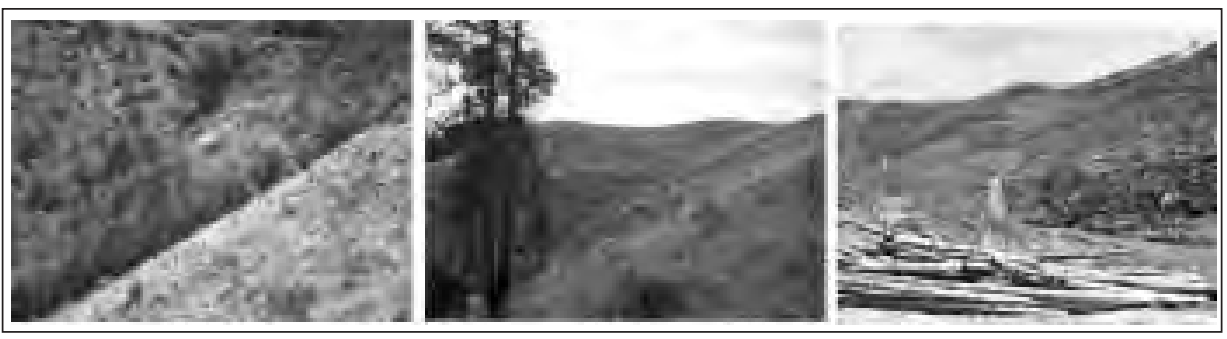

Figura 2. Vista general de la cuenca de El Real, apenas quemada en el incendio de 1994 (izda.), y de la de Alastuey (centro) afectada por el fuego, en proceso de regeneración. Trocha para la saca de madera en el barranco de Alastuey (dcha.). 
El incendio sucedido en agosto de 1994, tuvo una duración de 9 días y arrasó una extensión total de 1892 has. Se ha calificado a este incendio forestal como un "fuego de copas y de superficie", ya que afectó principalmente a las copas de los árboles, a matorrales y a pastos. Tras el fuego se inició la saca de madera (1994-1997), continuando con las labores de repoblación (1997-2000) en las que se aplicó un subsolado intermitente siguiendo líneas de máxima pendiente con una densidad de 1600 hoyos/ha. Esta técnica de repoblación intensifica una serie de problemas ligados a la erosión de suelo (Herrero Borgoñón y Rubio, 1994), que pueden contribuir a un aumento de la escorrentía superficial.

El incendio afectó a dos pequeñas cuencas adyacentes, Alastuey y El Real, de características ambientales semejantes (Tabla 1 y Fig. 3), pero con un diferente grado de afección por el fuego, que quemó el 75\% de la cuenca de Alastuey de 5,6 km² y el 33\% de la de El Real de $3,9 \mathrm{~km}^{2}$.

Tabla 1. Características ambientales de las cuencas analizadas.

\begin{tabular}{|l|l|l|}
\hline $\begin{array}{l}\text { CARACTERÍSTICAS } \\
\text { GENERALES }\end{array}$ & ALASTUEY & EL REAL \\
\hline Altimetría & $\begin{array}{l}\text { - Altura máxima: } 1.146 \mathrm{~m} \\
\text { - Altura mínima: } 690 \mathrm{~m}\end{array}$ & $\begin{array}{l}\text { - Altura máxima: } 1.547 \mathrm{~m} \\
- \text { Altura mínima: } 690 \mathrm{~m}\end{array}$ \\
\hline Pendiente media & $10,3 \%$ & $19,9 \%$ \\
\hline Litología & $89 \%$ de lutitas y areniscas & $\begin{array}{l}36,2 \% \text { de lutitas y areniscas } \\
\text { conglomerados en la parte } \\
\text { superior de la cuenca }\end{array}$ \\
\hline Vegetación & - Formaciones arbóreas (pino & $\begin{array}{l}\text { - Formaciones arbóreas } \\
\text { (haya, pino silvestre, } \\
\text { quejigo, ...) }\end{array}$ \\
\hline Superficie quemada quejigo). & - Matorrales y pastos & $33 \%$ \\
\hline
\end{tabular}




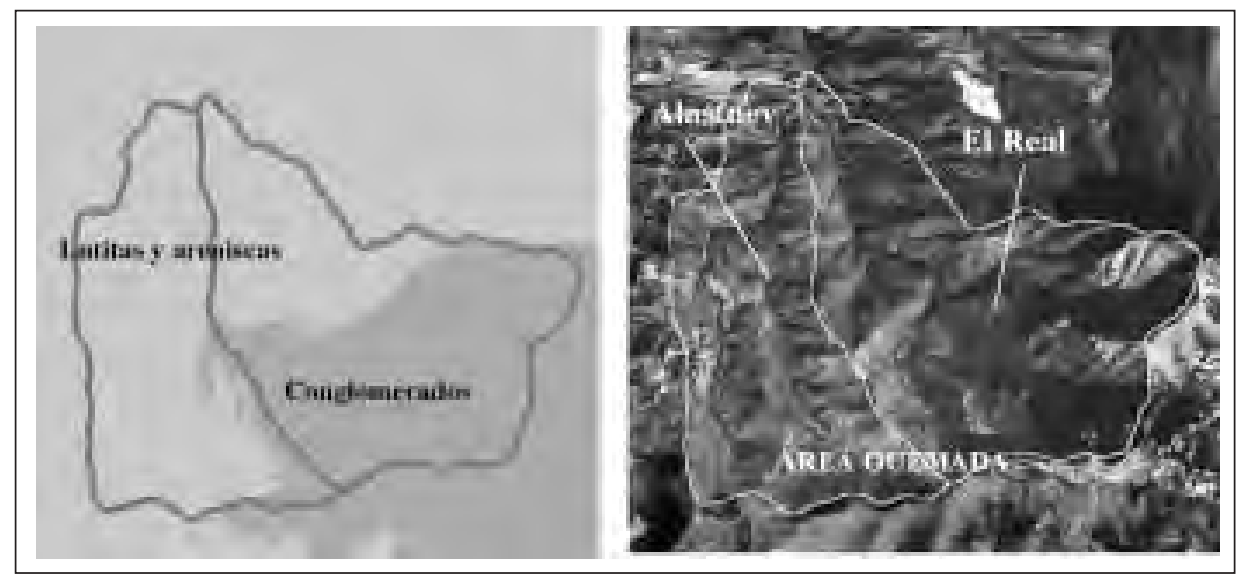

Figura 3. Composición litológica y distribución de las zonas quemadas en las cuencas.

\section{Metodología}

El análisis del comportamiento hidrológico de ambas cuencas se ha efectuado mediante el estudio de 11 crecidas, ocurridas entre el verano de 1997 y la primavera de 2000, durante las labores de saca de madera y repoblación posteriores al incendio. Se han analizado las precipitaciones, consideradas semejantes en las dos cuencas, y el caudal controlado por dos aforos localizados en la confluencia de ambos barrancos.

Con el objeto de caracterizar las entradas y salidas de agua se determinaron los siguientes atributos ligados a la precipitación correspondiente a cada una de las crecidas (resultados obtenidos a partir de la información cedida por el Instituto Nacional de Meteorología-Ministerio de Medio Ambiente): duración de la lluvia expresada en horas $\left(\mathrm{t}_{\mathrm{p}}\right)$, precipitación total en milímetros $(\mathrm{P})$, intensidad máxima en 1 hora $\left(\mathrm{P}_{1 \mathrm{~h}}\right.$ en $\left.\mathrm{mm}\right)$, precipitaciones caídas en las 6 y 24 horas anteriores a la crecida $\left(\mathrm{P}_{6 \mathrm{~h}}\right.$ y $\mathrm{P}_{24 \mathrm{~h}}$ en mm) y lluvia durante los 15 días $\left(\mathrm{P}_{15 \mathrm{~d}}\right.$, en $\left.\mathrm{mm}\right)$ y 30 días $\left(\mathrm{P}_{30 \mathrm{~d}}\right.$ en $\left.\mathrm{mm}\right)$ anteriores al aumento de caudal. Para la descripción de las crecidas se han utilizado las siguientes variables: tiempo de respuesta en horas $\left(t_{r}\right)$, caudal-adaptación total de la crecida $\left(Q_{t}\right.$, en $\left.1 / \mathrm{m}^{2}\right)$, caudal-aportación del pico de crecida $\left(\mathrm{Q}_{\mathrm{mx}}\right.$, en $\left.1 / \mathrm{m}^{2} / \mathrm{h}\right)$, coeficiente de escorrentía $(\mathrm{CE})$, descarga superficial $\left(\mathrm{Q}_{s}\right.$, en $\left.1 / \mathrm{m}^{2} / \mathrm{h}\right)$ y contribución de ésta a la descarga total $\left(\mathrm{Q}_{s} / \mathrm{Q}_{\mathrm{t}}\right)$.

En la primera exploración estadística se han calculado la media, la mediana, la desviación típica y los valores extremos de los datos en cada uno de los aforos y se han comparado (test $t$ de student). Igualmente, en función del número de crecidas y de la distribución no normalizada de los valores, se ha calculado el coeficiente de correlación Rho de Spearman, con el objeto de evaluar la influencia de las variables citadas en el comportamiento de cada una de las cuencas. Con los datos de caudal y precipitación se han elaborado los hidrogramas y pluviogramas correspondientes a las 11 crecidas, de los cuales se exponen algunos ejemplos (Fig. 5). 

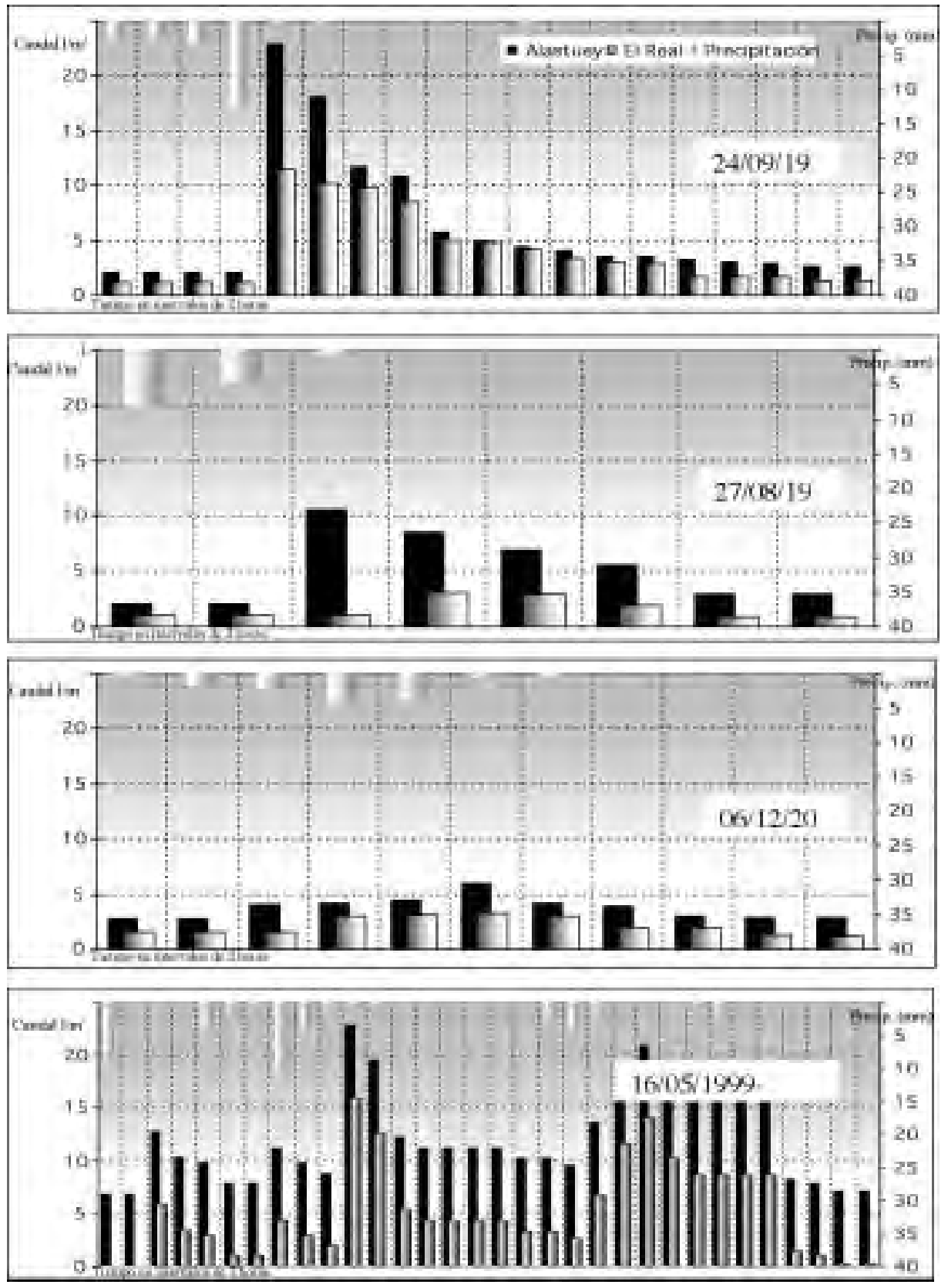

Figura 5. Ejemplos de hidrogramas correspondientes a picos de crecidas de las diferentes estaciones. 


\section{Resultados y discusión}

Los hidrogramas que representan las situaciones de crecida estacional (Fig. 5) muestran un comportamiento hidrológico diferente en ambas cuencas, siendo siempre más abundantes las respuestas del barranco de Alastuey (cuenca quemada).

Este comportamiento es especialmente evidente en la estación seca, verano y comienzos de otoño en la cuenca incendiada, con crecidas de tipo "pico", cuando la morfología del hidrograma se refleja como una fiel respuesta a precipitaciones aisladas y de carácter tormentoso. En estas condiciones el caudal del barranco de Alastuey puede "doblar" el de El Real durante el máximo de la crecida, aun con diferentes magnitudes como se observa en los hidrogramas de agosto y de finales de septiembre. Durante el invierno, los bajos caudales responden a un momento de frecuentes, pero escasas y poco intensas, precipitaciones que producen crecidas también mayores en la cuenca quemada, pero con una morfología más atenuada, carente de picos pronunciados, especialmente en el barranco de El Real. Por último, la primavera, máximo pluviométrico del área de estudio, arroja frecuentes precipitaciones de intensidad media, de tipo frontal, que provocan crecidas sucesivas, siempre mayores en la cuenca quemada, pero cuyas diferencias se van atenuando a medida que el suelo va estando más humectado y disminuye la capacidad de infiltración, especialmente en la cuenca boscosa de El Real.

Tabla 2. Estadística básica de las variables de las crecidas.

\begin{tabular}{|l|c|c|c|c|c|}
\hline Alastuey & Qt & Qmx & Qs & CE & tr \\
\hline Media & 1,462 & 0,384 & 0,192 & 10,009 & 3,82 \\
Mediana & 0,839 & 0,210 & 0,024 & 8,468 & 4,00 \\
Desv. típica & 1,934 & 0,481 & 0,448 & 6,990 & 0,60 \\
Mínimo & 0,309 & 0,077 & 0,008 & 3,462 & 2 \\
Máximo & 7,214 & 1,803 & 1,524 & 27,211 & 4 \\
\hline
\end{tabular}

\begin{tabular}{|l|c|c|c|c|c|}
\hline El Real & Qt & Qmx & Qs & CE & tr \\
\hline Media & 0,693 & 0,118 & 0,087 & 4,819 & 5,25 \\
Mediana & 0,378 & 0,070 & 0,002 & 3,956 & 6,00 \\
Desv. típica & 0,987 & 0,166 & 0,237 & 3,284 & 1,03 \\
Mínimo & 0,333 & 0,028 & 0,000 & 1,482 & 4 \\
Máximo & 3,662 & 0,610 & 0,802 & 12,461 & 6 \\
\hline
\end{tabular}


Tabla 3. Estadística básica de las precipitaciones.

\begin{tabular}{|l|c|c|c|c|c|c|c|}
\hline & P & Pmax1h & P6h & P24h & P15d & P30d & tp \\
\hline Media & 18,060 & 8,158 & 12,641 & 19,609 & 26,847 & 32,737 & 10,00 \\
Mediana & 12,330 & 5,859 & 8,170 & 13,632 & 22,804 & 29,804 & 6,00 \\
Desv. típica & 18,865 & 7,626 & 10,559 & 10,704 & 11,536 & 12,814 & 12,49 \\
Mínimo & 3,084 & 0,925 & 3,084 & 8,648 & 15,650 & 17,080 & 2 \\
Máximo & 68,219 & 27,000 & 32,937 & 39,937 & 48,120 & 53,120 & 46 \\
\hline
\end{tabular}

Tabla 4. Análisis comparativo de las variables hidrológicas.

\begin{tabular}{|l|c|c|c|c|c|}
\hline & Media & $\begin{array}{c}\text { Intervalo de } \\
\text { Confianza Inf. }\end{array}$ & $\begin{array}{c}\text { Intervalo de } \\
\text { Confianza Sup. }\end{array}$ & $\mathrm{t}$ & $\begin{array}{c}\text { Sig. } \\
\text { (bilateral) }\end{array}$ \\
\hline Qt & 0,768 & 0,114 & 1,422 & 2,618 & 0,026 \\
Qmx & 0,266 & 0,050 & 0,481 & 2,755 & 0,020 \\
Qs & 0,105 & $-0,038$ & 0,248 & 1,632 & 0,134 \\
Qs/Q & $-5,279$ & $-10,169$ & $-0,389$ & $-2,406$ & 0,037 \\
CE & 5,190 & 2,255 & 8,124 & 3,941 & 0,003 \\
$\operatorname{tr}$ & $-1,500$ & $-2,274$ & $-0,726$ & $-4,583$ & 0,003 \\
\hline
\end{tabular}

La estadística básica de los parámetros hidrológicos de las crecidas y el análisis comparativo entre cuencas se recoge en las Tablas 2 y 4, respectivamente. Asimismo la Tabla 3 muestra la descriptiva básica de las precipitaciones, consideradas las mismas en ambas cuencas dado su reducido tamaño y su contigüidad espacial.

En líneas generales, la comparación de los datos de descarga muestra diferencias estadísticamente significativas (Tabla 4). En términos de caudal, el registrado en la cuenca de Alastuey duplica al que se recoge en la cuenca de El Real. El tiempo de respuesta también es significativamente diferente: la cuenca de Alastuey aumenta su caudal rápidamente (3,82 h), mientras que la cuenca de El Real precisa de una media de 5,25 h para apreciar una subida en la curva de crecida. El coeficiente de escorrentía superficial es en la cuenca quemada de un $10 \%$, mientras que en cuenca no quemada alcanza tan sólo un $5 \%$. Estas diferencias a favor del barranco de Alastuey, quedan patentes a través de otras variables de descarga analizadas (caudal máximo en el pico de crecida, descarga superficial...). Al mismo tiempo destaca la mayor variabilidad de los datos hidrológicos en el barranco de Alastuey. 
Por otra parte, a pesar de la cautela interpretativa que exige el análisis de correlación entre los diferentes parámetros de precipitación y caudal, se pueden reseñar tres aspectos generales (Tabla 5).

En primer lugar, la escasa capacidad explicativa que tienen los parámetros de precipitación sobre los caudales en el caso de la cuenca de El Real (tan sólo es estadísticamente significativa la relación entre la descarga superficial y la precipitación previa en 24 horas). En segundo lugar, la importancia de la precipitación total (P) y de la intensidad máxima en 1 hora $\left(\mathrm{P}_{1 \mathrm{~h}}\right)$ a la hora de explicar el caudal total $\left(\mathrm{Q}_{\mathrm{t}}\right)$ y la descarga superficial $\left(\mathrm{Q}_{\mathrm{S}}\right)$ en la cuenca quemada. Finalmente, el tercer aspecto a destacar alude a la notable relación que existe entre la descarga superficial y la precipitación a medio y largo plazo (24 horas, 15 y 30 días) en la cuenca de Alastuey de donde se desprende la importancia del estado de humedad del suelo previo a la crecida.

Tabla 5. Coeficientes de correlación de Spearman de las variables analizadas en las cuencas. En negrita se indican las correlaciones significativas $(f \mathfrak{b}, 05)$.

\begin{tabular}{|c|c|c|c|c|}
\cline { 2 - 5 } \multicolumn{1}{c|}{} & \multicolumn{4}{c|}{ ALASTUEY } \\
\cline { 2 - 5 } \multicolumn{1}{c|}{} & Qt & Qmx & Qs & Qs/Qt \\
\hline $\mathrm{P}^{n}$ & $\mathbf{0 , 6 2 7}$ & 0,373 & 0,555 & 0,491 \\
$\mathrm{P}_{\mathrm{lh}}$ & 0,509 & 0,309 & $\mathbf{0 , 6 9 1}$ & $\mathbf{0 , 6 2 7}$ \\
$\mathrm{P}_{6 \mathrm{~h}}$ & 0,218 & 0,000 & 0,418 & 0,445 \\
$\mathrm{P}_{24 \mathrm{~h}}$ & 0,436 & 0,445 & $\mathbf{0 , 6 3 6}$ & $\mathbf{0 , 6 4 5}$ \\
$\mathrm{P}_{15 \mathrm{~d}}$ & 0,545 & 0,491 & $\mathbf{0 , 6 0 9}$ & 0,545 \\
$\mathrm{P}_{30 \mathrm{~d}}$ & 0,436 & 0,409 & $\mathbf{0 , 7 0 9}$ & $\mathbf{0 , 6 6 4}$ \\
\hline
\end{tabular}

\begin{tabular}{|c|c|c|c|}
\hline \multicolumn{4}{|c|}{ EL REAL } \\
\hline Qt & Qmx & Qs & Qs/Qt \\
\hline 0,327 & 0,318 & 0,456 & 0,316 \\
0,209 & 0,527 & 0,540 & 0,419 \\
0,164 & 0,218 & 0,321 & 0,181 \\
0,345 & 0,491 & $\mathbf{0 , 6 2 8}$ & 0,535 \\
0,245 & 0,382 & 0,521 & 0,400 \\
0,173 & 0,491 & 0,591 & 0,498 \\
\hline
\end{tabular}

La comparación de los hidrogramas y de las variables relativas a las crecidas de ambas cuencas muestra diferencias claras en su comportamiento hidrológico.

La contribución de la escorrentía directa es considerablemente mayor en la cuenca quemada de Alastuey donde la escorrentía superficial supera a la de El Real. Las crestas o picos de crecida son más marcados en el caso de Alastuey que en El Real, hasta el punto de que alguna crecida sólo se refleja en el barranco de la cuenca quemada; este hecho es más evidente en verano, estación en la que la precipitación registrada es de carácter tormentoso, produciéndose una escorrentía superficial que responde a un flujo de tipo hortoniano.

En el barranco de Alastuey, se puede observar una respuesta hidrológica directa con respecto a las precipitaciones, ya que su caudal depende de la lluvia registrada, demos- 
trando que la capacidad de infiltración de la cuenca es muy limitada. Ejercicios de simulación de lluvia en microparcelas sobre la superficie quemada (Pérez-Cabello et al., 2003b) arrojaron una tasa de infiltración por debajo del 50\%, mientras que en la parcela control los valores superaban el $95 \%$, en la línea de los resultados obtenidos por Terry et al. (1994) en el centro-norte de Portugal y sumándose al aumento de los coeficientes de escorrentía -entre 2 y 21 veces superiores a la situación anterior al fuego-, reconocidos por otros autores a escala de parcela en ámbitos distintos, como el chaparral californiano (De Bano et al., 1976), los bosques del Montseny y de la Sierra de Prades (Sala y Calvo, 1990), el NW español (Soto et al., 1994) o la sierra valenciana (Andreu et al., 1994). La interceptación, en relación con el menor grado de cubrimiento vegetal, es bastante baja al igual que la capacidad de infiltración del suelo, hecho éste relacionado con la pérdida de cubierta vegetal, la decapitación de los horizontes superficiales del suelo, la reducción de la cantidad de materia orgánica y la compactación de la superficie edáfica ligada a las labores de repoblación. Por lo tanto, la respuesta hidrológica de la cuenca de Alastuey se rige esencialmente por los procesos de generación de escorrentía superficial que explican que la descarga superficial sea más alta que en la cuenca de El Real.

La evolución de las crecidas en el barranco de El Real es significativamente diferente, ya que esta cuenca muestra un comportamiento hidrológico más complejo, en el que los hidrogramas reflejan más tímidamente la dinámica de las precipitaciones. La respuesta a la lluvia es más retardada que en el caso de Alastuey y claramente amortiguada. El aumento de caudal está condicionado por procesos de interceptación e infiltración, resultantes de una cubierta vegetal más densa y de un suelo que absorbe más agua, lo cual explica una menor aportación de la descarga superficial al caudal total.

\section{Agradecimientos}

La investigación realizada en las cuencas de Alastuey y El Real se ha efectuado con el apoyo económico del premio "Félix de Azara", concedida por la Diputación Provincial de Huesca el año 2004 y del Proyecto "Efectos erosivos del fuego a lo largo de un gradiente climático. Aportaciones para la gestión de áreas quemadas" (REN200200133/GLO).

\section{Referencias bibliográficas}

Andreu, V., Rubio, J.L., FortezA, J y CERni, R. (1994). Long term effects of forest fires on soil erosion and nutrient losses. En: Soil erosion as a consequence of forest fires (Sala, M. y Rubio J.L. Ed.) Geoforma Ediciones, Logroño, 79-91.

BISSONNAIS, L. Y. (1996). Aggregate stability and assessment of soil crustability and erodibility: theory and methodology. Eur. J. Soil Sci. S47, 425-437. 
Calvo-Cases, A. y CerdÀ, A. (1994). An example of the changes in the hydrological and erosional response of soil after a forest fire, Pedralba (Valencia), Spain. En: Soil erosion as a consequence of forest fires (Sala, M. y Rubio J.L. Ed.) Geoforma Ediciones, Logroño, 99-110.

Cerdà, A. Imeson, A.C. y CAlvo, A. (1995). Fire and aspect induced differences on the erodibility and hydrology of soils at La Costera, Valencia, southest Spain. Catena, 24, 4: 289-304.

Coehlo, C.O., Shakesby, R.A., Walsh, R.D., Terry, J. y Ferreira, A. (1990). Responses of surface and sub-surface soil water movement and soil erosion to forest fires in Eucalyptus globulus and Pinus pinaster forest, Agueda basin. Proceedings of International Conference on Forest Fire Research, Coimbra.

DE BANO, L.F. (1971). The effect of hydrophobic substances on water movement in soil during infiltration, Proc.-Soil Sci. Soc. Am., 35: 340-343.

De Bano, L.F., Mann, L.D. y Hamilton, D. A. (1970). Translocation of hydrophobic substances into soil by burning organic litter, Proc.-Soil Sci. Soc. Am., 34: 130-133.

De BANO, L.F., Rice, R.M. y ConRAD, C.E. (1979). Soil heating in Chaparral fires: effects on soil properties, plant nutrients, erosion and run-off. Res. Paper PSW-145. Forest Service. USDA.

DíAZ-Fierros, F., BENITO, E. y PÉREZ, R. (1987). Evaluation of the USLE for the prediction of erosion in burnt forest areas in Galicia (NW Spain). Catena, 14: 189-199.

DíAz-Fierros, F. y Rubio, J.L. (1990). Metodologías de estudio de los procesos de erosión hídrica del suelo en España, Cuad. Área Cienc. Agrar., 12.

Fernández, S., Marquínez, J. y Menéndez-Duarte, R. (2005). A susceptibility model for post wildfire soil erosion in a temperate oceaic mountain area of Spain. Catena, 61, 2-3: 256-272,

Giovannini, G. (1994). The effect of fire on soil quality. En: Soil erosion as a consequence of forest fire (Sala, M. y Rubio J.L. Ed.) Geoforma Ediciones. Logroño,15-27.

GiOvanNinI, G. y LuCCHESI, S. (1983). Effect of fire on hydrophobic and cementing substances of soil aggregates. Soil Science, 136: 231-236.

Kutiel, P., Lavee, H., Segev, M. y Benyamini, Y. (1995). The effect of fire-induced surface heterogeneity on rainfall-runoff-erosion relationships in an eastern Mediterranean ecosystem, Israel. Catena, 25, 1-4: 77-87.

MARQUÉS, M.A. y MORA, E. (1992). The influence of aspect on runoff and soil loss in a Mediterranean burnt forest. Spain. Catena, 19, 3-4: 333-344.

MARTíneZ-FERnÁNDEZ, J. y DíAZ-PEREIRA, E. (1994). Changes of the physical and chemical properties in a soil affected by forest fire in Sierra Larga (Murcia). En: Soil erosion as a consequence of forest fires (Sala, M. y Rubio J.L. Ed.) Geoforma Ediciones. Logroño, 67-78. 
Molina, M. J., García-Fayos, P. y SAnroque, P. (1994). Short-term changes on aggregate stability and organic matter content after forest fire in a calcareous soil in Valencia. En: Soil erosion as a consequence of forest fires (Sala, M. y Rubio J.L. Ed.) Geoforma Ediciones. Logroño, 43-52.

MoODY, J.A. y MARTIN, D. A. (2001a). Initial hydrologic and geomorphic response following a wildfire in the Colorado Front Range. Earth Surface Processes and Landforms, 26: 1049-1070.

Moody, J.A. y MARTin, D. A. (2001b). Post-fire, rainfall intensity-peak discharge relations for three mountainous watersheds in the western USA. Hydrological Processes, 15: 2981-2993.

Pérez-CABello, F., EcheVerríA, Ma T., IbARrA, P. y DE la Riva, J. (2002). Estudio experimental de la dinámica ambiental postincendio en el Prepirineo oscense: el incendio de Agüero. En: Aportaciones a la Geomorfología de España en el inicio del Tercer Milenio (Pérez-González, A., Vegas, J. y Machado, M. Ed.). Madrid, 307-31.

Pérez-Cabello, F., EcheverríA, Ma T., Ibarra, P. y DE la Riva, J. (2003a). Alteraciones edáficas en comunidades vegetales quemadas 15 años tras el fuego. En: Control de la erosión y degradación del suelo (Bienes, R. y Marqués, M.J., Ed.) Madrid, 211-214.

Pérez-Cabello, F., SÁnchez de Mena, Mª J., Echeverría, M ${ }^{\mathrm{a}}$ T. y Ibarra, P. (2003b). Comportamiento hidrogeomorfológico de un bosque quemado y sometido a labores de repoblación. El incendio de San Juan de la Peña (Huesca, España). En: Landnutzungswandel und Landdegadation in Spanien (Marzoloff, I., Ries, J., de la Riva, J. y Seeger, M., Eds.). Universität Frankfurt am Main y Universidad de Zaragoza, 85-97.

Sala, M. y CAlvo, A. (1990). Response of four different mediterranean vegetation types of runoff and erosion. En: Vegetation and erosion (Thornes, J.B., Ed.). John Wiley, 348-362.

Sala, M. y Rubio, J.L. (Ed.) (1994). Soil erosion as a consequence of forest fires. Geoforma Ediciones, Logroño, 275.

SÁnchez , J.R., MANGAS, V.J., ORTIZ, C. y Bellot, J. (1994). Forest fire affect on soil chemical properties and runoff. En: Soil erosion as a consequence of forest fires (Sala, M. y Rubio J.L. Ed.) Geoforma Ediciones. Logroño, 53-65.

SEEGER, M y BEGUERÍA, S. (2003). La respuesta hidrológica en dos cuencas experimentales con diferentes usos del suelo. En: Landnutzungswandel und Landdegadation in Spanien (Marzoloff, I., Ries, J., de la Riva, J. y Seeger, M. Eds.). Universität Frankfurt am Main y Universidad de Zaragoza, 203-221.

SHAKESBY, R.A., y DOERR, S.H. (2006). Wildfire as an hydrological and geomorphological agent. Earth Science Reviews, 74: 269-307.

Soler, M. y SAlA, M. (1992). Effects of fire and of clearing in a Mediterranean Quercus ilex woodland: an experimental approach. Catena, 19: 321-332. 
Soler, M., Sala, M. y Gallart, F. (1994). Post fire evolution of runoff and erosion during an eighteen month period. En: Soil erosion as a consequence of forest fires (Sala, M. y Rubio J.L. Ed.). Geoforma Ediciones. Logroño, 149-161.

Soto, B., Basanta, R., Benito, E., Pérez, R. y Díaz Fierros, F. (1994). Runoff and erosion from burnt soils in Northwest Spain. En: Soil erosion as a consequence of forest fires (Sala, M. y Rubio J.L. Ed.) Geoforma Ediciones, Logroño, 91-98.

TERRY, J.P. (1994). Soil loss from erosion plots of differing post fire cover, Portugal. En: Soil erosion as a consequence of forest fires (Sala, M. y Rubio J.L. Ed.) Geoforma Ediciones. Logroño,133-148.

Trabaud, L. (1981). Man and fire: impacts on Mediterranean vegetation En: Mediterranean-Type Shrublands (di Castri, F., Goodall, D.W. y Specht, R.L., Ed.). Elsevier, Amsterdam, 523-537.

Úbeda, X. y SAla, M. (1996). Cambios en la física del suelo e incremento de la escorrentía y la erosión tras un incendio forestal. Actas IV Reunión de Geomorfología, S.E.G., 559-572.

ÚbedA, X. y SALA, M. (1998). Variation in runoff and erosion in three areas with different fire intensities. Geoökodynamik, XIX , 179-188. 